\title{
Congrès Québécois de la Recherche Clinique en Sciences Neurologiques
}

\author{
Les abrégés du congrès qui a eu lieu les 4 et 5 mai 1984 \\ à Montréal, Québec
}

1.

Callosotomie anterieure dans les epilepsies frontales

J.M. Saint-Hilaire, N. Giard, G. Bouvier, et R. Labrecque, (Montréal, Québec)

L'investigation des épilepsies frontales en vue d'un traitement chirurgical, est un probleme très complexe. Nous avons étudié 17 cas d'épilepsie frontale au moyen d'électrodes implantées. Dans 14 cas, nous avons déterminé la zone épileptogène au moyen des crises spontanées et de la stimulation électrique suivies d'une résection corticale. Dans 3 autres cas, il ne nous a pas été possible de déterminer quel lobe frontal était d'abord pris par la décharge et nous avons conclu qu'il s'agissait d'épilepsie frontale.

Deux cas semblables ont été soumis à une callosotomie antérieure. Après plus d'un an, il persiste une réduction remarquable du nombre de crises, également une amélioration du comportement des malades. Un troisième cas présentant des foyers indépendants bilatéraux dans les deux lobes frontaux a été opéré il y a plus d'un an avec des bons résultats.

II semble que le mécanisme de contrôle des crises consiste en un blocage de la généralisation de la décharge épileptique à travers le corps calleux antérieur tel que nous le démontrerons par des EEG pré et post-callosotomie. Dans ces épilepsies bifrontales, l'atteinte simultanée bilaterale des lobes frontaux semble responsable de l'apparition des manifestations cliniques.

La callosotomie antérieure se révèle une solution valable pour certains cas d'épilepsie frontale sévère chez lesquels une résection corticale est impossible.

2.

L'investigation des epilepsies frontales au moyen d'electrodes en profonduer

F. Delisle, J.M. Saint-Hilaire, F. Veilleux, G. Bouvier, N. Giard, J. Montplaisir, et R. Labrecque. (Montréal, Québec)

La valeur localisatrice de crises spontanées enregistrées au moyen d'électrodes en profondeur pour l'identification des foyers épileptiques est maintenant acceptée de façon générale.

Nous avons procédé à une étude rétrospective de 16 malades présentant une épilepsie frontale qui ont subi une cortectomie (13/16) ou une callosotomie antérieure (3/16) depuis 1977 avec un follow-up moyen de 2 ans. Nous avons analysé la valeur localisatrice de l'examen neurologique, des manifestations éileptiques cliniques enregistrées en circuit fermé de télévision, de l'investigation neuroradiologique, de l'EEG sur le scalp et de l'EEG enregistré en profondeur.
Les résultats chirurgicaux ont été évalués selon les critères de Crandall: pour la cortectomie, $7 / 13$ des cas se situent dans le groupe $A, 6 / 13$ dans le groupe $B$ et pour la callosotomie antérieure les 3 cas sont dans le groupe B.

On remarque:

1) qu'en général, l'examen neurologique et l'investigation neuroradiologique sont relativement moins localisateurs;

2) que l'EEG ictal sur le scalp n'a pas de valeur mais que les anomalies intercritiques EEG sur le scalp ou en profondeur ont une légère valeur;

3) que les manifestations cliniques critiques ont une valeur supérieure;

4) que la stimulation électrique ne donne pas de faux positif et aide à localiser le foyer si les EEG de profondeur ne sont pas suffisamment localisateurs;

5) que des lésions pathologiques ont été trouvées dans 5 cas. Elles ne président pas un meilleur résultat.

6) qu'il y a une nette prépondérance de foyers dans le gyrus cingulaire et dans l'aire motrice supplémentaire comparativement aux foyers touchant les régions frontales externes.

3.

Phénytoine et muscimol

Robert Lalonde et M.I. Botez (Montréal, Québec)

La phénytoine (PHT) est injectée ( $25 \mathrm{mg} / \mathrm{kg}$ ) une fois par jour pendant 20 jours consécutifs chez les rats mâles de la souche Sprague-Dawley $(n=10)$. Lors des jours 21 et 28 , ces rats reçoivent un dosage d'un agoniste des récepteurs du GABA, le muscimol ( $2 \mathrm{mg} / \mathrm{kg})$ qui suffit pour produire le comportement de catalespie. La catalepsie est mesurée selon quatre tests différents. Le test de l'akinésie a comme mesure, le temps avant l'initiation du premier mouvement du rat dans un champs ouvert. Le test de la barre et le test de la grille ont comme mesure le temps avant le déplacement du rat de sa position initiale. Finalement une mesure de la résistance à la poussée de main est employée. Dans tous les tests, le muscimol augmente les latences et cause une plus grande résistance à la poussée de main.

Les résultats indiquent que le retrait de la PHT après administration chronique cause une sous-sensibilité du comportement de catalepsie induite par le muscimol, lors du jour 21 mais non du jour 28 . Il est proposé que l'administration chronique de la PHT produit une soussensibilité des récepteurs du muscimol.

Dans une deuxième expérience, nous avons trouvé que le comportement de catalepsie induit par le muscimol n'est pas inhibé par la bicuculline, un antagoniste des récepteurs GABA-A. Ce résultat suggère que l'administration chronique de la PHT produit une sous-sensibilité d'un type de récepteurs du GABA non susceptible à la bicuculline.

(Travail subventionné par la Fondation Jeanne-Mance). 
4.

Un protocole original d'evaluation de la memoire durant le test a l'amytal

I. Rouleau, R. Labrecque, J.M. Saint-Hilaire, N. Giard et G. Bouvier (Montréal, Québec)

Afin de prévenir d'éventuels troubles amnésique suite à une lobectomie temporale, il est essentiel d'évaluer les capacités de l'hippocampe qui devra assurer seul le fonctionnement amnésique suit à la chirurgie.

Nous avons élaborer un protocole original qui permet, parl'utilisation d'une tâche de reconnaissance non-verbale, de contourner le problème de l'aphasie résultant d'une injection gauche et, qui est applicable lorsqu'il y a encore à l'EEG, un fort pourcentage d'ondes lentes. Cette méthode permet un comparaison directe entre les performances observées après anesthésie de l'hémisphère droit et gauche chez un même patient.

La majorité des protocoles existants évalue la rétention de matériel acquis sous l'effet de l'Amytal seulement lorsque le cerveau a retrouvé son fonctionnement normal. Notre protocole nous permet de dissocier et de comparer l'acquisition et le rappel avant, durant et après l'anesthésie d'un hémisphère. Les profils amnésiques résultants sont évalués en fonction du site épileptogène.

La validité interne du protocole a pu être démontrée, chez 2 patients, par l'obtention de résultats identiques après répétition du test avec le même hémisphère.

Aucun des 20 patients opérés n'a développé de trouble amnésique à l'exception des déficits sub-cliniques déjà décrits par Brenda Milner.

\section{5.}

\section{Severe encephalopathy in Cree infants belonging to a large} kindred in Chisasibi

Deborah N. Black, E. Andermann, T. Costa, C. Dumont, M.E. Kabay, P. Kaplan, R. Nagy, E. Reece, K.M. Villemure, G.V. Watters (Montréal, Québec)

We report 17 Cree Indian infants in seven closely related sibships. Group I (eight patients) abruptly developed seizures, vomiting, stupor, and spasticity between three and a half and nine months, during a febrile upper respiratory illness or, in one patient, following a typhoid immunization. This was succeeded by blindness, fixed pupils, absent tendon reflexes, and death from cardiorespiratory arrest or status epilepticus 10 days to 14 months after onset. CSF showed lymphocytic pleocytosis in one case; protein 58-320 mg\%; CSF IgG was elevated (3.9-5.6 $\mathrm{mg} \%$ ). One patient had a severe combined immunodeficiency. Diffuse sudanophilic degeneration of white matter was present at autopsy.

Group II (nine patients) showed progressive microcephaly, quadriparesis, mental retardation and recurrent infections from birth. There was CSF lymphocytic pleocytosis, elevation of protein, and elevation of IgG (maximum $25 \mathrm{mg} \%$ ). Autopsy showed perivascular lymphocytic cuffing, calcifications and demyelinated zones throughout the white matter. One patient showed changes resembling both groups.

Normal biochemical studies in several patients have ruled out the known metabolic diseases of the CNS. Adenovirus or CMV has been grown from brain, CSF, or urine in some cases. These two clusters of disease may be attributed to a single autosomal recessive gene, to two independently segregating genes, or to environmental factors interacting with genetic subsceptibility to atypical CNS infection in this highly inbred group.

\section{6.}

Traitement avec la L-carnitine chez un patient atteint d'acidémie propionique

B. Lemieux, R. Giguère. C. Auray-Blais. T. Nawar, (Sherbrooke. Québec)

Il est possible de corriger certains troubles enzymatiques conduisant à une acidémie organique à l'aide de cofacteurs comme la biotine pour les déficiences multicarboxylases ou la vitamine $\mathrm{B} 12$ pour les formes répondantes d'acidémie méthylmalonique. Pour les patients souffrant de déficience enzymatique isolée telle qu'une déficience en propionyl CoA carboxylase (P.C.C.) le correctif est plus difficile à apporter. Nous avons cependant obtenu des résultats très encourageants avec un traitement à la $\mathrm{L}$-carnitine chez une fille de 10 mois, avec une déficience en P.C.C.

Il s'agit d'une fille unique, née d'une grossesse et d'un accouchement normaux avec un poids de 7 livres et 9 onces dont la diathèse familiale est négative. Sa croissance et son développement ont été normaux jusqu'à deux mois sauf la présence d'épisodes hebdomadaires de troubles digestifs sous forme de vomissement "de 24 heures" et de refus d'alimentation. A l'âge de deux mois elle fut admise avec un tableau suggestif de syndrome hémolytique urémique avec acétonurie. Par la suite, elle présenta une stagnation psycho-motrice et pondérale avec troubles paroxystiques mal définis et hypotonie généralisée. A quatre mois, à la suite d'une infection des voies respiratoires supérieures avec anorexie et irritabilité, elle fut admise dans un état d'acidose métabolique (pH à 6,9, trou anionique à 29). Le diagnostic fut confirmé par chromatographie en phase gazeuse et spectrométrie de masse (urine et sang) nous rélévant de grandes quantités d'acides organiques comme l'acide méthylcitrique, l'acide hydracrylique, l'acide $\beta-O H$-valérique, l'acide 3-OH-propionique, la propionylglycine, la tiglylglycine, l'acide 3-OH-méthylbutyrique: patron consistant avec une acidémie propionique. Aucune activité enzymatique de la P.C.C. ne fut notée sur la culture de fibroblaste.

L'étude de la L-carnitine nous a permis de déceler:

1) un rapport acylcarnitine et carnitine libre très élevé, typique à l'acidémie propionique.

2) présence d'une déficience secondaire en L-carnitine.

Au régime hypotrotidique initial plus ou moins efficace, nous avons ajouté un traitement à la $\mathrm{L}$-carnitine: $100 \mathrm{mg} / \mathrm{kg} /$ jour puis $125 \mathrm{mg} / \mathrm{kg}$ jour. Cette enfante a répondu rapidement à la $L$-carnitine par une augmentation de sa tolérance aux protéines, un gain de poids, une normalisation progressive du tonus musculaire et du développement psycho-moteur. Il n'y a pas eu d'autres épisodes d'acidose métabolique avec ou sans hyperammoninémie.

Cette thérapie peut potentiellement servir pour d'autres acidémies organiques telles que: acidémie méthylmalonique, acidémie glutarique et isovalérique, ainsi que dans d'autres conditions telles que le syndrome de Reye,

7.

\section{Profil urinaire d'un enfant atteint de fucosidose Type II}

N.M.K. NG Ying Kin, R. Giguère, B. Lemieux (Montréal, Sherbrooke, Québec)

La fucosidose est une maladie de surcharge, transmise de façon autosomale récessive, qui atteint le système nerveux. Cliniquement, on peut distinguer au moins deux phénotypes - Type I et Type II selon la présence ou non des lésions cutanées et la progression des symptômes neurologiques. Bien que le type I soit classé forme sévère et le Type II forme moins sévère ou chronique, cette différenciation n'est pas toujours adéquate. L'étude clinique, histologique et biochimique d'un enfant atteint de fucosidose type II nous permet de discuter le profil urinaire dans cette maladie lysosomale.

Il s'agit d'un fils unique dont les parents sont consanguins, né à terme, d'une grossesse et d'un accouchement normaux avec un poids de 5 livres et 11 onces. La croissance et le développement ont été normaux jusqu'à vers l'âge de trois mois, où l'on note progressivement l'apparition de troubles digestifs, respiratoires et infectieux nécessitant de multiples hospitalisations pour retard staturo-pondéral, otite, anémie allergie et carence maternelie. A 17 mois, il présente un Denver de 10 mois, un test de sueur négatif et un EEG normal. Par la suite, jusqu'à l'âge de trois ans, on assiste à une stagnation puis régression des acquisitions psycho-motrices incluant le langage, et on note une surdité partielle, une hépatomégalie et un léger dysmorphisme avec signes pyramidaux progressifs. Une investigation détaillée incluant la recherche des cytosomes dans la biopsie de la peau et de la conjonctive et une chromatographie des oligosaccharides nous permettent de nous orienter vers un diagnostic de fucosidose. Vers l'âge de 5 ans, on constate la présence d'une angiokératose diffuse ainsi que des lésions rétiniennes 
cliniques et neurophysiologiques. Le patient, actuellement âgé de 11 ans, montre une régression lente, importante, psychomotrice avec retard staturo-pondéral, hypotrophie et signes pyramidaux progressifs. L'étude histo-pathologique à la microscopie électronique des biopsies (peau, rein, nerf, muscle, muqueuse rectale et conjonctive) montre la présence d'inclusions claires et foncées tel que décrit dans cette maladie lysosomale.

Sur le plan biochimique, une déficience de l'enzyme lysosomale, alpha-L-fucosidase, fut constatée dans les fibroblastes et leucocytes du patient, confirmant ainsi le diagnostic de fucosidose. Cependant, cette déficience enzymatique ne nous permettait pas de différencier entre les deux phénotypes de cette maladie. par contre, l'analyse par chromatographies sur couche mince et haute pression liquide des glyco-conjugués, exrétés excessivement dans l'urine, nous a été fort utile dans ce contexte. Ainsi, chez notre patient, il y avait une plus grande quantité de glycopeptides mais très peu d'oligosaccharides libres qu'on trouvait en majeure partie dans l'urine du Type I. Une analyse plus approfondie de ces glycopeptides et d'autres glyco-conjugués, notamment les glycolipides, devrait nous permettre de mieux comprendre la pathophysiologie de différents phénotypes de cette maladie et aussi nous en donner un marquer biochimique.

\section{8.}

Adénomes envahissants de l'hypophyse: Etude pathologique et clinique

\section{Françoise Robert (Montréal, Québec)}

L'adénome envahissant est un adénome qui dépasse le dure-mère de la loge sellaire pour infiltrer les structures voisines: cerveau, sinus caverneux et os sphénoïde.

Notre étude porte sur 41 cas ( $7 \%$ de tous les adénomes hypophysaires étudiés en pathologie a l'hôpital Notre-Dame).

Sur le plan pathologique la destruction et l'infiltration tumorale du plancher sellaire avec extension dans le sinus sphénoïdal sont présentes dans $92 \%$ des cas. L'extension supradiaphragmatique médiane est retrouvée dans $66 \%$ des cas. Le lobe temporal (22\% des cas), le tronc cérébral ( $20 \%$ des cas) et le lobe frontal ( $10 \%$ des cas) peuvent être envahis. Dans 2 cas où le tronc cérébral était infiltré il y a eu dissémination tumorale dans l'espace sous-arachnoïdien ( $5 \%$ des cas). L'invasion unilatérale du sinus caverneux est présente dans $37 \%$ des cas tandis que dans $20 \%$ des cas elle est bilatérale. par contre elle a été retrouvée dans tous les 13 cas étudiés à l'autopsie.

Sur le plan clinique le seul signe spécifique est la rhinorrhée spontanée (12\% des cas). Des signes neurologiques d'atteinte du sinus caverneux n'ont été observés que dans $12 \%$ des cas.

Chez 4 malades le décès a été directement causé par le caractère envahissant de l'adénome.

\section{9.}

\section{Bilateral hemianopia and headache}

\section{S. Gosselin and D. Boghen (Montréal, Québec)}

Sudden onset of acute blindness is a rare event. It may result from lesions of either the anterior or the posterior visual pathway. Anteriorly it occurs in pituitary apoplexy and simultaneous anterior ischemic optic neuropathy secondary to temporal arteritis. Posteriorly it is a consequence of occipital lobe disease. Cerebral blindness may be due to anoxia and infarction of the occipital lobes. It has also been described following seizures, head trauma and arteriography. In none of these situations is cerebral blindness likely to be accompanied by severe headache. The presence of severe headache in this setting suggests the diagnosis of complicated migraine. The following case indicates that a subarachnoid hemorrhage is another important diagnostic consideration. A similar case has not been previously reported. A 64 year old female presented with a history of abrupt nocturnal onset of severe bifrontal headache and complete loss of vision. Three hours after the onset of headache, she had a brief generalized seizure. When first seen in neurology an hour after the seizure, she was well awake but with slowed mentation. Vision was reduced to light perception in both eyes. The pupils were equal and reacted normally to light. Funduscopic examination and ocular motility were normal. There was no neck stiffness. Neurological examination was otherwise unremarkable. A brain CT-scan without contrast was normal. Twenty-four hours later, there was persistent headache. Visual fields were confined to a small central island of vision in both eyes. A lumbar puncture on the day of admission yielded blood-tinged cerebrospinal fluid containing 3000 red cells per cubic millimeter. Angiography showed an aneurysm of the posterior communicating artery and spasm of all the cerebral vessels on the right side. Visual fields remained unchanged until ten days later when Goldmann perimetry showed normal visual fields. A craniotomy was performed and confirmed the existence of the aneurysm which showed signs of recent bleeding.

10.

\section{Sclérose en plaques: familles à cas multiples}

\section{P. Duquette, J. Pleines, L. Charest, L. Savard (Montréal, Québec)}

Le rôle d'une prédisposition génétique à la S.E.P. est l'objet de nombreuses recherches. L'incidence familiale de la S.E.P. est del'ordre de $15 \%$. De nombreuses études de familles à cas multiples ont été rapportées. Elles observent la répartition des antigènes HLA chez les membres atteints et les membres non atteints. La plupart concluent a un certain degré de ségrégation des haplotypes chez les sujets atteints.

Nous rapportons nos résultats à propos de 9 familles à cas multiples (familles nucléaires), dont certaines comptent un nombre élevé de frères-soeurs atteints. Nous avons examiné cliniquement tous les membres disponibles et nous avons pu leur faire des P.L. et étudier le L.C.R. chez les mêmes individus. Ceci nous a permis d'identifier des sujets cliniquement sains, ou avec des syndromes neurologiques très discrets, porteurs des anomalies biologiques de la S.E.P. dans le L.C.R..

Si on tient compte uniquement des paires de frères-soeurs ("sibpairs") avec S.E.P. clinique, la répartition observée des haplotypes ne differe pas du nombre attendu. Par contre, si on inclut les sujets avec une "S.E.P. biochimique", la répartition observée des haplotypes diffère nettement du nombre attendu. Les implications de ces résultats seront discutees.

\section{1.}

Étude des potentiels évoqués auditifs chez des enfants traités pour hypothyroïdie.

Emmanuèle Laureau, Michel Vanasse, Jacques Letarte (Montréal, Québec)

Nous avons étudié les potentiels évoqués auditifs (P.E.A.) chez vingt et un (21) patients âgés de 5 à 9 ans, porteurs d'hypothyroïdie congénitale, sous traitement substitutif.

Nous avons retrouvé des anomalies dans 13 cas sur 21 :

- 10 enfants montrent un engourdissement des latences ou délais: le délai III - V est dimunié pour 8 d'entre eux, et il existe un raccourcissement du délai I-V chez 7 sujets.

-3 enfants présentent un allongement de la latence de l'onde I mais avec un délai I-V normal.

En se basant sur les données de la littérature concernant les P.E.A. chez les hypothyroïdiens non traités et les hyperthyroïdiens, on peut émettre l'hypothèse que la dimunution du délai I-V ou temps de conduction centrale, pourrait être un indice de surimprégnation de l'hormone thyroïdienne. Ceci concorde avec une élévation du taux de $T_{4}$ qui a été effectivement retrouvée dans 10 cas sur 21 .

Quant à l'allongement de la latence de l'onde 1, il s'agit d'une atteinte auditive périphérique dont on ne peut être certain qu'elle est reliée à l'hypothyroïdie.

Des corrélations seront faites éventuellement avec les données biologiques et psychologiques dont nous disposons pour ces 21 sujets. Il sera possible de préciser dans l'avenir l'intérêt de l'étude des P.E.A. en terme diagnostic, pronostic et thérapeutique chez les enfants traités pour hypothyroïdie congénitale. 
12.

Étude des potentiels évoqués somesthésiques précoces chez des enfants traités pour hypothyroïdie.

Emmanuèle Laureau, Michel Vanasse, Jacques Letarte (Montréal, Québec)

Nous avons étudié les potentiels évoqués somesthésiques précoces (P.E.S.) et les vitesses de conduction nerveuse chez neuf (9) patients âgés de 5 à 9 ans, porteurs d'hy pothyrö̈die congénitale et sous traitement substitutif.

Nous avons retrouvé des anomalies dans 7 cas, portant essentiellement sur des allongements de latence et délais:

- chez 4 enfants nous avons noté un allongement des intervalles $N_{13}$ $-N_{19}$.

- un allongement de l'intervalle $\mathrm{N}_{19}-\mathrm{P}_{22}$ chez 6 patients.

Chez 7 des patients étudiés, nous avons pu comparer le résultat des études de potentiels évoqués avec le résultat obtenu par des évaluations neuro-psychologiques détaillées. Il semble y exister une corrélation très nette entre celle-ci et les intervalles $\mathrm{N}_{19}-\mathrm{P}_{22}$. En effet, les trois (3) enfants dont l'évaluation neuro-psychologique est normale représente un intervalle $\mathrm{N}_{19} \mathrm{P}_{22}$ normal. Par contre les quatre (4) enfants présentant des déficiences au niveau de leur évaluation neuro-psychologique présentent une augmentation de ce délais ou intervalle $\mathrm{N}_{19}-\mathrm{P}_{22}$.

Etant donné qu'il n'existe aucune donnée ni chez l'animal ni chez I'humain concernant les P.E.S. dans I'hypothyroïdie, il est difficile d'évaluer la signification précise de cette anomalie en terme anatomique ou physiologique. Des études subséquentes nous permettrons certainement de préciser l'intérêt des P.E.S. en terme diagnostic, pronostic et thérapeutique chez les enfants présentant une hypothyroıdie congenitale.

13.

Aspects de la prépondérance féminine dans la S.E.P.

P. Duquette, J. Pleines, L. Charest (Montréal, Québec)

La prépondérance féminine dans la distribution de la S.E.P. selon les sexes est bien établie, mais mal expliquée,. Nous allons présenter des données qui semblent indiquer qu'il y a des différences, du moins sur le plan global, entre la S.E.P. chez les femmes et la S.E.P. chez les hommes.

Ces données ont trait:

à l'âge de début,

aux formes précoces,

à la distribution des antigènes HLA,

à la concordance des jumeaux,

à certains aspects évolutifs,

à des aspects clinques,

Nous tenterons d'en tirer quelques conclusions.

14.

\section{Déficit de représentation imagée.}

M.I. Botez, Mireille Olivier, Jean-Lorrain Vézina, Thérèse Botez et Benjamin Kaufman (Montréal, Québec; Cleveland, Ohio)

On présente l'observation d'un patient de 38 ans gaucher qui a une déficience dans l'imagerie visuelle depuis sa naissance. Les autres signes et symptômes étaient: un léger déficit dans la mémoire topographique, une légère négligence spatiale unilatérale droite, écriture en miroir et perte de la capacité de rêver. Le patient présentait une hypoplasie de l'hémisphère droit ainsi qu'un déficit anatomo-fonctionnel de la portion postérieure du corps calleux, qui ont été mis en évidence par l'artériographie, par la tomodensitométrie du cerveau et par la résonnance nucléaire magnétique. On considère que le déficit dans l'imagerie visuelle est secondaire à un syndrome de dysconnection partiel. Chez ce patient il y avait une dissociation nette entre un excellente pensée cognitive (le QI global était à 54) par rapport au déficit évident dans la représentation imagée. Une classification nouvelle des cas publiés de perte de l'imagerie visuelle sera envisagée. En ayant comme point de départ les deux étapes de développement de la pensée spatiale chez l'enfant tel que décrites par Piaget, les auteurs montrent que dans les formes pures de défaut ou de perte de l'imagerie visuelle (2-3 cas dans la litterature) on peut observer que la notion de l'espace sensori-moteur est préservé tandis que la représentation imagé est perdue ou sérieusement déficitaire; il s'agit d'une dissolution dans le sens jacksonien qui peut être observée chez ces patients.

15.

Frontal lobe epilepsy syndrome: Clinical, EEG and pathological correlation

L.F. Quesney, P. Gloor, F. Andermann, A. Olivier and C. Krieger (Montréal, Québec)

This study attempts to correlate the most relevant clinical, EEG and pathological findings in 28 patients (males 22 , females 6) presenting with long-standing history of poorly controlled frontal lobe seizures. Partial frontal lobectomy under electrocorticography monitoring was performed in 27 patients.

Extensive pre-operative seizure monitoring was performed in all patients. Five patients underwent EEG exploration with chronically implanted depth electrodes in both frontal and temporal lobes.

Results: The clinical presentation was characterized by numerous brief-lasting seizures usually not preceded by warning. The clinical seizure pattern was multiple including: a) partial motor tonic or clonic seizures with frequent loss of consciousness; b) high incidence of automatism (50-60\% of patients) posing a differential diagnosis with partial complex seizures of temporal lobe origin. Adequate localization of the epileptic disturbance was obtained by means of nasoethmoidal and supraorbital electrodes.

16.

Lesions multiples non metastatiques a la T.O. du crâne

J.G. Blain, J.L. Léger, J. Lamoureux (Montréal, Québec)

L'espoir que la tomodensitométrie du crâne faciliterait le diagnostic des tumeurs cérébrales doit être reconsidéré.

Nous avons revu les t.o. du crâne de 106 malades consécutifs où un diagnostic de métastase cérébrale a été posé. Ces diagnostics avaient été posés sur la base d'une multiplicité des lésions, la présence de lésions bien délimitées associées à un oedème et la présence de lésions qui s'éliminent après injection de contraste.

Chez 14 malades, soit $12 \%$ de nos cas, la preuve d'une lésion non métastatique fut faite. Une tumeur cérébrale primitive fut démontrée soit par craniotomie ou à l'autopsie chez 5 malades. Trois malades souffraient d'une maladie vasculaire, un malade souffrait d'une méningite aux cryptocoques avec formation d'abcès. Les autres pathologies retrouvées furent un lymphome, un malade avec maladie démyélinisante et un hamartome cérébral.

Les indicateurs pouvant distinguer entre des lésions multiples secondaires à une maladie néoplasique métastatique et une atteinte cérébrale primitive seront discutés.

17.

Evaluation neurologique standardisée dans les accidents cérébrovasculaires aiguës.

R. Côté, V.C. Hachinski, B. Shurvell, J.W. Norris (Montréal, Québec)

Même si les accidents cérébrovasculaires constituent une des causes les plus importantes de morbidité et mortalité neurologique en Amérique du Nord, il n'existe présentement aucun système d'examen clinique, standardisé et fiable afin d'évaluer ces patients dans la phase aiguë. Notre but est de présenter un tel système et les résultats préliminaires sur sa fiabilité clinique. Ce système comprend dix items, tous les 
patients sont évalués avec les trois premiers soit: 1) Niveau de conscience, 2) Orientation 3) Language. Les items restant peuvent être regroupés en deux sections dépendant de la présence ou non de problèmes de compréhension. Si un patient ne présente aucun problème de compréhension il sera évalué avec la section $A_{1}$, qui comprend: 1) Motricité faciale, 2) Motricité proximale membre supérieur, 3) Motricité distale membre supérieur, 4) Motricité membre inférieur. Au contraire si un problème de compréhension existe la section $A_{2}$ sera utilisée afin de définir la réponse motrice au niveau: 1) Visage, 2) Membres supérieurs, 3) Membres inférieurs. Chaque item est ensuite gradué selon la sévérité de déficit neurologique. Ce système fut évalué chez un groupe de 33 patients avec des déficits neurologiques divers. Les patients furent examinés par 3 ou 4 observateurs soit 2 neurologues et 2 infirmières. L'analyse statistique des premières 110 évaluations montra un haut degré de consistence interne (alpha 90\%) et également un très bon agrément entre observateurs (Kappa).

Ce système standardisé pourrait se révéler utile afin de: 1) Détecter precocement des variations dans l'état neurologique, 2) Servir comme méthode d'évaluation objective, 3) Permettre l'uniformité des données cliniques dans les études multicentriques.

\section{8.}

\section{Preliminary report on the effectiveness of prostacyclin in stroke}

\section{A.M. Hakim, R.P. Pokrupa and L.S. Wolfe (Montréal, Québec)}

Prostacyclin $\left(\mathrm{PGI}_{2}\right)$ has been proposed as a drug with therapeutic potential in ischemic conditions. We are presently evaluating its effectiveness in patients with cerebrovascular accidents. The results of the initial open-label trial in 5 patients are reported here. An additional 30 patients are being studied double-blind against placebo.

$\mathrm{PGI}_{2}$ infusion was started within $\mathbf{4 8}$ hours from the onset of clinical deficits in 5 patients. They were evaluated clinically and by routine biochemistry and hematology as well as CT scans both before and at intervals during and after $\mathrm{PGI}_{2}$ infusion. Exclusion criteria included coma, severe hypertension or diabetes, a significant medical or neurological disorder other than the stroke, recent surgery and risk of hemorrhage, or presence of blood on the CT. $\mathrm{PGI}_{2}$ was infused intravenously 8 hours daily for 5 consecutive days starting at 2 and gradually increasing up to $10 \mathrm{ng} / \mathrm{kg} / \mathrm{min}$ depending on tolerance.

Prostacyclin was in general well tolerated. Flushing was noted in all patients, 2/5 had vascular-type headaches, and all showed a mild asymptomatic drop in blood pressure. By the end of infusion, 3/5 patients showed moderate clinical improvement and $2 / 5$ had no clinical change, but at one month $2 / 5$ showed complete resolution and $3 / 5$ moderate improvement in their deficit. The results of the double-blind study should permit more reliable assessment of this drug's effectiveness following a stroke.

\section{9}

Potentiels évoqués auditifs dans les pathologies du tronc cérébral.

\section{J.J. Dufour, Y. Comair, G. Filion (Montréal, Québec)}

Nous présentons ici notre expérience sur 5 ans dans l'utilisation des potentiels évoqués dans les lésions de l'angle ponto-cérébelleux et du tronc cérébral. Trente-six cas de neurinôme de l'acoustique prouvés ont été investigués; un allongement de l'intervalle I-III a été trouvé dans $38 \%$. L'audition était bien conservé chez ces patients. Dans les cas où l'audition était très atteinte, l'enregistrement de toutes les ondes s'avère beaucoup plus difficile. Un allongement de l'onde $V$ ou de l'intervalle I-V est rapporté dans $55 \%$. Un allongement de l'intervalle III.V homo ou contralatéral observé dans $5 \%$ indiquait une compression du T.C. et suggérait un élément de mauvais pronostic.

Douze cas de SEP avec atteinte du T.C. ont été évalués. Des anomalies unilatérales manifestées par une diminution de l'amplitude ou un allongement III-V ont été observées dans $73 \%$. Dans $23 \%$ ces anomalies étaient bilatérales et un seul faux négatif a été enregistré. Deux familles atteintes de SEP ont aussi été évaluées. Des anomalies ont été enregistrées seulement chez les membres qui ont présenté des attaques de sclérose en plaques et l'importance des anomalies était en relation directe avec la sévérité des lésions démyélinisantes.

Huit cas de tumeur du T.C. ou du cervelet avec extension au T.C. ont été investigués. Les potentiels évoqués auditifs nous ont permis un diagnostic exact de latéralité dans tous les cas. De plus, la mesure des divers interval les nous a permis d'apprécier l'extension de la tumeur au niveau du tronc cérébral.

Notre expérience confirme la place bien établié des potentiels évoqués auditifs dans les lésions de l'angle ponto-cérébelleux et du tronc cérébral.

\section{0.}

\section{Modifications du transport endothélial et astrocytaire des acides aminés neutres en epilepsie expérimentale}

\section{Y. Robitaille, K. Kemball, J. Gotman (Montréal, Québec)}

Les acides aminés neutres (AAN) de faible poids moléculaire sont transportés "in vitro" par les cellules endothéliales cérébrales a l'aide d'un système à polarisation abluminal dont le substrat-type est l'A lanine (Système "A", Oxenford et Christensen, 1967). Son isomère B montre une prédominance de captation astrocytaire à haute affinité en tissu cérébral normal, très compétitive avec le GABA et qui ne peut s'intégrer à la synthèse protéique à cause de sa structure moléculaire. De nombreuses modifications de ce système ont été observées sur des cicatrices gliales induites par microinjections $(5 \mu \mathrm{l}$.) de chlorure ferrique au niveau du cortex moteur de rats Sprague-Dawley dans le but de reproduire un modèle d'épilepsie focale post-traumatique humaine, tel que décrit par Willmore et coll. Des augmentations marqués de la captation comparativement aux spécimens normaux (p.001) ont été observées par morphométrie ultrastructurale 5,30 et 120 jours post-induction, surtout au niveau des pieds astrocytaires périvasculaires. Des augmentations semblables par les cellules endothéliales furent observées qui indiquèrent un changement de polarité vers le flanc luminal des profils de captation de $B$-Alanine tritiee au niveau des marges cicatricielles. Ces expériences furent reprises "in vivo" par injection I.P. de ${ }^{14} \mathrm{C}$ - $\beta$-Alanine $(125 \mu \mathrm{Ci} / \mathrm{kg})$ et la captation évaluée par densitométrie. L'évaluation statistique révéla un profil de captation significatif dans les régions cicatricielles correspondant à la topographie des astrocytes réactifs 15 jours post-induction malgré l'absence de Bleu Evans dans ces mêmes régions après perfusion I.V., qui indiquait que la barrière Hémoencéphalique (BHE) s'était déjà reconstituée. L'utilisation de l'acide 3-Mercaptopropionique, un agent convulsif inhibiteur de la synthèse du GABA injecté I.P. ( $30 \mathrm{mg} / \mathrm{kg}$ ) et suivi du traceur dès l'atteinte du seuil convulsif, provoqua un recrutement marqué d'une captation de forte densité à pré-dominance thalamique bilatérale et corticale s'étendant maintenant à toute la convexité ipsilatérale à la lésion. Ces résultats supportent l'hypothèse que la régulation de la captation de B-Alanine exogène par les astrocytes réactifs est liée aux concentrations de GABA endogène. La reconstitution précoce de la BHE souligne que cette captation est aussi dépendante d'un synergisme endothélioglial impliquant sélectivement les AAN de faible poids moléculaire dans ce contexte expérimental, puisque le ${ }^{14} \mathrm{C}$-L-Acide Glutamique ne put reproduire ces résultats dans les mêmes conditions.

21.

Pharmacokinétique du B.C.N.U. et caractéristiques métaboliques des gliomes malins.

J.G. Villemure, L. Yamamoto, M. Diksic, J. Théron, W. Feindel (Montréal, Québec)

Certains aspects métaboliques des gliomes malins furent analysés in vivo à l'aide de la tomographie à émission de Positron. Avec cette technique, nous avons obtenu chez des patients porteurs de tumeurs cérébrales malignes, des études de débit sanguin régional (cCBF), du métabolisme régional de l'oxygène (rCMR02) et du métabolisme régional du glucose (rCMRGI).

Les résultats démontrent que le débit sanguin régional et le métabolisme du glucose sont habituellement en parallèle et reflètent l'activité cellulaire 
de la tumeur: ces paramètres sont diminués dans les portions moins actives des tumeurs, comme dans la nécrose. A la périphérie de la tumeur, dans les zones édémateuses, il y a diminution du débit sanguin et aussi de l'utilisation du glucose. Il existe une dissociation entre le degré d'utilisation de l'oxygène et du glucose: en effet, pour une forte utilisation de glucose, on note une faible utilisation d'oxygène, suggérant un métabolisme, une glycolyse anaérobique.

L'étiquetage radioactif du B.C.N.U. nous a permis, à l'aide de la tomographie à émission de positron, d'établir in vivo, chez des patients porteurs de tumeur cérébrale maligne, la pharmacokinétique de cet agent chémothérapeutique.

Les concentrations, distributions du B.C.N.U. sont ainsi déterminées au site tumoral, à la région péri-tumorale ou au cerveau normal. Ces analyses furent obtenues chez des patients ayant reçu par voie intraartérielle carotidienne.

Le protocole employé actuellement pour la chémothérapie des gliomes à grade élevé est discuté.

\section{2.}

Influence du mannitol intraveineux sur la perméabilité de la barrière hémato-tumorale cérébrale.

\section{J.G. Villemure, L. Yamamoto, W. Feindel (Montréal, Québec)}

La barrière hémato-tumorale est un facteur restrictif dans la pénétration de certains agents chémothérapeutiques au niveau de pathologies cérébrales, soit infectieuses ou néoplastiques. A l'aide de la Tomographie à émission de Positron, nous avons analysé quantitatjvement, l'influence du Mannitol intraveineux sur la perméabilité de la barrière hématotumorale chez 18 patients porteurs de tumeur cérébrale primaire. L'étude fut pratiquée à l'aide du Gallium 68 administré par voie intraveineuse. Des 18 cas analysés, il y avait 14 astrocytomes grade 3 ou 4 et 4 astrocytomes grade 2 . Les résultats démontrent une augmentation de la perméabilité au site tumoral avec infusion de Mannitol. Chez 11 des 14 gliomes à grade élevé, l'augmentation de la perméabilité était de l'ordre de $65 \%$. Chez les tumeurs à grade bas, l'augmentation était de $25 \%$. Avec la technique d'administration intraveineuse, il n'y a pas eu de changement de perméabilité observé au cerveau normal.

Cette étude démontre que l'administration intraveineuse de Mannitol en concentration de $500 \mathrm{mg} / \mathrm{kg}$ augmente la perméabilité de la barrière hémato-tumorale de façon sélective au site tumoral et à la zone marginale, sans affecter la barrière normale. Cette augmentation de la perméabilité se retrouve davantage au niveau des gliomes malins. L'administration d'un agent oncolytique, pourrait vraisemblablement augmenter la concentration de l'agent chémothérapeutique à la tumeur, de façon sélective, sans augmenter les risques de toxicité cérébrale ou systémique.

\section{3}

Interêt de la biopsie musculaire dans le diagnostic differentiel de l'arthrogrypose congénitale multiple.

Elizabeth Strehl, Michel Vanasse, Pierre Brochu (Montréal, Québec)

Entre 1979 et 1983, nous avons évalué 22 bébés présentant une arthrogrypose congénitale multiple. 21 de ces enfants ont eu soit un électromyogramme ( $18 \mathrm{cas}$ ) ou une étude histo-chimique du muscle ( 17 cas). 14 ont eu les deux. Chez les enfants ayant eu une étude histochimique du muscle, 15 spécimens ont été obtenus par biopsie à l'aiguille alors que chez 2 enfants l'étude histo-enzymologique a été faite à partir de prélèvements d'autopsie.

Chez 9 de ces bébés, nous avons retrouvé soit à l'E.M.G. et/ou à la biopsie musculaire des anomalies de type myopathique, souvent d'allure dystrophique. Ceci représente une proportion étonnamment élevée par rapport à ce qui est connu dans la littérature. Chez 10 autres enfants, l'étiologie de ce problème était neurogène. L'anomalie neurogène était d'origine spinale chez 4 patients, d'origine cérébrale chez 3 et mixte dans les trois autres cas. Enfin, dans les autres cas évalués, un présentait un syndrome malformatif héréditaire et chez deux bébés l'étiologie demeure imprécise puisque l'investigation s'est avêrée négative.
Compte tenu de ces résultats, nous croyons que la biopsie musculaire devrait-être incluse dans le bilan de base de l'arthrogrypose congénitale multiple car elle permet d'en mieux préciser l'étiologie, le pronostic et l'aspect génétique.

\section{4.}

Obstruction des voies aériennes supérieures dans les maladies extra-pyramidales.

S. Gauthier, W. Vinckne, R. Dollfus, R. Hanson, M.G. Cosio (Montréal, Québec)

27 patients avec maladie de Parkinson idiopathique, paralysie supranucléaire progressive ou tremblement essentiel ont subi des tests de fonctions respiratoires avec attention particulière à la courbe flotvolume. Celle-ci s'est révélée anormale chez 24 patients, avec (1) des oscillations régulières, synchrones avec le tremblement des membres $(4-8 \mathrm{~Hz})$, ou (2) des oscillations irrégulières. La visualisation directe des cordes vocales par endoscopie a démontré que ces oscillations correspondent à des mouvements rythmiques ou irréguliers des cordes vocales et des structures supra-glottiques, avec occlusion intermittente de la glotte. 12/23 patients ont amélioré leurs flots respiratoires en respirant un mélange d'hélium et d'oxygène, démontrant une obstruction physiologique des voies respiratories supérieures. 4 autres eurent besoin d'assistance respiratoire. Les patients les plus à risque selon notre étude sont ceux souffrant de dyskinésies induites par des agents dopaminergiques. De plus, la sévérité des anomalies pulmonaires augmente avec le stade (1 à 5 ) de la maladie de Parkinson. Une obstruction des voies respiratoires supérieures doit être soupçonnée en cas de détresse respiratoire aïgue ou intermittente chez les patients souffrant de maladies extra-pyramidales, avec traitement approprié.

25.

Complications neurologiques périphériques de la maladie vasculaire aorto-iliaque.

M.L. D’Amour, L.H. Lebrun, A. Rabbat et Jules Trudel (Montréal, Québec)

Les manifestations cliniques de la maladie vasculaire aorto-iliaque sont bien connues. Les complications neurologiques périphériques qui peuvent en découler sont rarement décrites dans la littérature et la corrélation entre l'atteinte neurologique et vasculaire y est peu élaborée.

Au cours des dernières années, nous avons eu la possibilité d'examiner six (6) malades avec une atteinte neurologique périphérique directement reliée à une maladie vasculaire aorto-iliaque ou à sa chirurgie. Chacun des malades a été examiné par neurologue et a subi un électromyogramme.

Nous avons trouvé des atteintes du plexus lombo-sacré et des mononévrites. Nous avons tenté par la suite d'établir une corrélation entre la lésion vasculaire et neurologique. Pour ce faire, l'anatomie vasculaire et neurologique périphérique des régions impliquées sera revue, commentée et leur interrelation discutée.

Cette étude nous permet de conclure à une relation étroite entre les structures vasculaires aorto-iliaques et le système nerveux périphérique du membre inférieur.

Cette relation permet d'expliquer certaines pathologies nerveuses périphériques.

26.

Le syndrome d'hemiplegie alternante paroxystique dans l'enfance

M.L. Saint-Hilaire, F. Andermann, K. Silver, N. Morris (Montreal, Québec)

Ce syndrome décrit par Verret et Steele, est une entité clinique qui est souvent confondue avec l'épilepsie. Vingt-deux cas ont déja été décrits, et nous présentons 3 autres cas. Les aspects caractéristiques de ce syndrome sont un début avant l'âge de 18 mois, des attaques 
fréquentes d'hémiplégie alternante, précédées d'irritabilité et de pleurs. Des anomalies des mouvements oculaires, du nystagmus, de la dystonie, de la choroathéthose, et une dysfonction autonomique (troubles respiratoires, tachycardie, mydriase, diaphorèse) font aussi partie des attaques qui durent le plus souvent quelques heures. Le pronostic est similaire chez tous le patients décrits dans la littérature et chez nos 3 patients, avec l'apparition de déficits neurologiques incluant un retard intellectuel et de langage, une hypotonie axiale et des mouvements involontaires. Un de nos patients ainsi qu'un patient de Verret et Steele, continuent à présenter des attaques après un follow up de 18 ans. Une étiologie migraineuse est suspectée depuis le début mais est encore controversée. Les mères de nos 3 patients souffrent de migraine. Bien que le traitement antimigraineux soit en général inefficace, une prophylaxie antimigraineuse agressive avec le propranolol et la pizotyline, semble modifier la durée et l'intensité des attaques chez 2 de nos malades. Une de nos patientes est soumise de plus à un essai de Nimodipine.

27.

Encéphalomyélite subaiguë reliée au SIDA: Observation clinicopathologique de trois cas pédiatriques.

Jean Michaud, Guy Geoffroy, Elizabeth Rousseau, Normand Lapointe et Jean Joncas (Montréal, Québec)

La population pédiatrique est un sous-groupe pouvant présenter le syndrome d'immunodéficience acquise (SIDA). Les paramètres cliniques et immunologiques sont similaires à ceux observés chez les adultes mais les complications nuerologiques sont encore mal connues. Dans notre centre, parmi les cinq enfants décédés du SIDA, trois ont présenté un tableau clinique et des changements morphologiques similaires. Ces enfants, âgés de 7 à 58 mois ont présenté, à des degrés variables, des signes de spasticité des extrémités avec hyperréflexie et hypotonie axiale. Le cas atteint le plus sévèrement s'était d'abord présenté avec un tableau de myélite transverse et a évolué vers une quadriparésie spastique. Un cas dont l'atteinte clinique était moins sévère s'est légèrement amélioré peu avant son décès. Dans les trois cas, des changements mineurs et non spécifiques ont parfois été retrouvés dans le LCR, à l'EEG ou à la tomographie axiale. L'examen neuropathologique a démontré, dans les trois cas, des changements d'encéphalomyélite subaiguë intéressant surtout la moelle épinière et le tronc cérébral. La sévérité des changements reflétait la sévérité de l'atteinte clinique et sa durée d'évolution avec, dans un cas, une atteinte diffuse de tout le système nerveux central. Les changements inflammatoires sont associés à une démyélinisation non spécifique mais significative. La revue de la littérature nous indique que ce tableau morphologique a été retrouvé chez des adultes porteurs de SIDA mais la présentation clinique est en partie différente. Ce tableau n'a pas été rapporté chez les enfants. Il est probablement le résultat d'une atteinte virale directe mais un mécanisme de type "allergique" est possiblement associe.

28.

Des régions cérébrales différentes peuvent être à l'origine de crises d'épilepsie en mangeant. Etude à partir de 13 patients.

Guy Rémillard, Frédérick Andermann, Allan Sherwin, André Olivier, Théodore Rasmussen, Jim Rowan, Allan Guberman, Georges Patry, Santiago Jiménez-Roldan (Montréal, New York, Ottawa, Québec, Madrid)

Les crises survenant en mangeant peuvent être soustendues par de l'activité épileptique au niveau du lobe temporal ( 9 patients). Cette activité critique se situait au niveau de la région péri-amygdalienne chez un patient guéri après une lobectomie temporale incluant l'amygdale et laissant I'hippocampe en place. Les voies afférentes demeurent inconnues chez ce groupe de patients.

Les crises survenant en mangeant peuvent aussi être soustendues par de l'activité épileptique au niveau de structures extratemporales (4 patients). Elles persistent après une lobectomie temporale et hémisphérectomie modifiée excluant les gyri antérieur et postérieur au gyri pré et post centraux chez un patient. La localisation électrographique intercritique, la symptomatologie plus élémentaire sensitive ou motrice des crises et les tentatives chez 3 des 4 patients de ce groupe de changer certaines caractéristiques sensitives des aliments pour prévenir les crises, suggèrent une implication des voies thalamo-corticales dans l'élaboration des afférences sensitives. 